\title{
Coordination for Networks of Dynamic Agents with Time-Varying Delays
}

\author{
Hongwang Yu ${ }^{1}$, Baoshan Zhang ${ }^{1}$, Yufan Zheng ${ }^{2}$ \\ ${ }^{1}$ School of Mathematics and Statistic, Nanjing Audit University, Nanjing, China \\ ${ }^{2}$ Department of Mathematics, Shanghai University, Shangha, China \\ E-mail: $\{$ yuhongwang, zbs\}@nau.edu.cn, \{yfzheng, yuhw05820107\}@shu.edu.cn \\ Received November 21, 2009; revised December 5, 2009; accepted December 7, 2009
}

\begin{abstract}
This paper is devoted to the study of the coordinate stability in undirected networks of dynamical agents with time-varying transmission delay. Neighbor-based rules are adopted to realize local control strategies for these continuous-time autonomous agents. Sufficient and necessary conditions in terms of linear matrix inequalities (LMIs) are given to guarantee the coordination of dynamical agents. Numerical simulations are given and demonstrate that our theoretical results are effective.
\end{abstract}

Keywords: Multi-Agent System, Time-Varying Delay, Consensus Protocol, Laplacian

\section{Introduction}

The coordinate stability of multi-agent systems is an important research topic in engineering applications, including moving in formation for fleets of unmanned aerial vehicles (UAVs), satellite clusters and mobile sensor networks. In the last few years, it has attracted more attention in diverse fields in physics, biophysics, systems biology, applied mathematics, mechanics, computer science and control theory.

In the multi-agent systems, agents are usually coupled and interconnected with some simple rules including a proposed first-order/two-order dynamical model and nearest-neighbor rules. Using graph theory, Jadbabaie et al. [1] provided a theoretical explanation for the consensus behavior of dynamical multi-agents. The extended results under some more relaxable conditions are studied in [2]. Saber $e t$ al. investigated a systematical framework of consensus problem under a variety of assumptions on the network topology (fixed or switching), presence or lack of communication delays, and directed or undirected network information flow $[3,4]$.

In networks of the dynamic agents, time-varying delays may arise naturally, e.g., because of the moving of the agents, the congestion of the communication channels, the asymmetry of interactions, and the finite transmission speed due to the physical characteristics of the medium transmitting the information. The different consensus protocols have been investigated in [4-6], where the communication delay is a fixed constant. The average-consensus problem of agents under continuous-time networks with both switching topology and time-delay is studied in $[7,8]$, where the dynamics order of each agent is one. A leader-following consensus problem for multiple agent with communication transmission time delays is discussed in [9], where the dynamics of each agent is second order.

Motivated by [7] and [9], we study the coordinated stability of multi-agent systems where the dynamics of each agent is second order in this paper. The communication transmission time delays of multi-agent systems are varying and the interconnection graph of the agents is undirected. The method used in this paper is partly motivated by the work of $[10,11]$.

This paper is organized as follows. In Section 2, we recall some properties of graph and give the problem formulation. Coordinated stability analysis of the agents under network is given in Section 3. Section 4 gives a simulation example. Section 5 is a conclusion.

\section{Preliminaries}

By $G=(V, E, A)$, we denote an undirected graph with an weighted adjacency matrix $A=\left[a_{i j}\right]$, where $V=\left\{p_{1}, p_{2}, \mathrm{~L}, p_{M}\right\}$ is the set of nodes, $E \subseteq V \times V$ is the set of edges. The node indexes belong to a finite index set $\underline{M} @\{1,2, \mathrm{~L}, M\}$. An edge of $G$ is denoted by $e_{i j}=\left(p_{j}, p_{i}\right)$ for some $i, j \in \underline{M}$. The adjacency elements $a_{i j}$ are defined in following way: $e_{i j} \in E \Leftrightarrow a_{i j}>0$ 
and $e_{i j} \notin E \Leftrightarrow a_{i j}=0$. Moreover, we assume $a_{i i}=0$ for all $i \in \underline{M}$. The set of neighbors of node $p_{i}$ is denoted by $N_{i}=\left\{p_{j} \in V \mid\left(p_{i}, p_{j}\right) \in E\right\}$.

A diagonal matrix $D=\left\{d_{1}, \mathrm{~L}, d_{M}\right\} \in R^{M \times M}$ is a degree matrix of $G$, whose diagonal elements $d_{i}=\sum_{j=1}^{M} a_{i j}$ for $i \in \underline{M}$. Then the Laplacian of the weighted graph $G$ is defined as $L=D-A$. A graph is called connected if there exists a path between any two distinct vertices of the graph.

Lemma 1 The graph $G$ with the Laplacian $\mathrm{L}$ is connected if and only if $\operatorname{rank}(\mathrm{L})=\mathrm{M}-1$ and all eigenvalues of $\mathrm{L}$ are of positive real numbers except that only one eigenvalue is zero with eigenvector $1_{M}=(1, \mathrm{~L}, 1)^{T}$.

In this paper, we consider a network of dynamical agents defined by a connected graph $G=(V, E, A)$. The node set $V$ consists of dynamical agents $p_{i}, i \in \underline{M}$. The dynamics of $p_{i}, i \in \underline{M}$ are identical and described as follows.

$$
\begin{gathered}
\hat{\alpha}=v_{i} \\
m_{i} \underset{\alpha}{\alpha}=k v_{i}+u_{i} \\
y_{i}=F\left(\begin{array}{l}
x_{i} \\
v_{i}
\end{array}\right)
\end{gathered}
$$

where $x_{i} \in R^{n}$ is the location vector of agent $p_{i}$, $v_{i} \in R^{n}$ represents its velocity vector of the $i$-th agent, $u_{i} \in R^{n}$ is its coupling inputs and $m_{i} \in R^{n}$ is its mass. The control gain $k$ is designed later. The output map indicates that the state information of dynamical agents is measured by, for example, some remote sensor and transmitted to other agents in network [10].

Due to time-delay in communicated network, the control protocol of the dynamical agent $p_{i}$ is a neighborbased linear control law in the form that

$$
u_{i}=\sum_{p_{j} \in N_{i}} a_{i j}\left(y_{j}\left(t-\tau_{i j}(t)\right)-y_{i}\left(t-\tau_{i j}(t)\right)\right)
$$

where $N_{i}$ is the set of neighbors of agent $p_{i}$ and $a_{i j}$ are adjacency elements of $A$. The $\tau_{i j}(t) \geq 0$, denoting the communication transmission time-delay from agent $p_{j}$ to agent $p_{i}$. In the following, we assume that time-varying delays in (2) satisfy

$$
0 \leq \tau_{i j}(t) \leq d, \quad \tau_{i j}^{\alpha}(t) \leq h
$$

or

$$
0 \leq \tau_{i j}(t) \leq d
$$

For $t \geq 0$. That is to say, nothing has been known about the derivative of $\tau_{i j}(t)$, where $d$ and $h$ are posi- tive constant numbers.

To focus our study in a main stream, we simply assume that $m_{i}=1$, the observation matrix $F=$ $\left[\begin{array}{ll}I_{n \times n} & 0_{n \times n}\end{array}\right]$ and $\tau_{i j}(t)=\tau(t)$ for all $i, j \in M$ in this paper. We shall give the conditions, under which the network of dynamical agents ( $\operatorname{ref}\{$ dyn0 $\}$ ) achieve asymptotical consensus stability meaning that there exists a fixed position (equilibrium) $x^{*} \in R$ such that for $i \in M$

$$
\begin{aligned}
& \lim _{t \rightarrow \infty} x_{i}(t)=x^{*} \otimes 1_{n} \\
& \lim _{t \rightarrow \infty} v_{i}(t)=0_{n \times 1}
\end{aligned}
$$

\section{Coordination of Dynamic Agents with Time-Varying Delay}

We study the collective behavior of dynamical agents under a class of communicated networks. The collective behavior of dynamical agents in network can be described by $x(t)=\left(x_{1}^{T}(t), \mathrm{L}, x_{M}^{T}(t)\right)^{T} \in R^{M n}, \quad v(t)=$ $\left(v_{1}^{T}(t), \mathrm{L}, v_{M}^{T}(t)\right)^{T}$ and its communication topology is characterized by a connected graph $G$. Вy $x(0)=\left(x_{1}^{T}(0), \mathrm{L}, x_{M}^{T}(0)\right)^{T}, v(0)=\left(v_{1}^{T}(0), \mathrm{L}, v_{M}^{T}(0)\right)^{T}$, we denote the initial locations and the initial velocities of the agents, respectively.

\subsection{Description of Dynamic Systems}

Under control protocol (2) with $\tau_{i j}(t)=\tau(t)$ for all $i, j \in \underline{M}$, the dynamical equations of each agent of multi-agent systems are written by

$$
\xi \xi_{i}(t)=A \xi_{i}(t)+B \sum_{p_{j} \in N_{i}} a_{i j}\left(\xi_{j}(t-\tau(t))-\xi_{i}(t-\tau(t))\right)
$$

where $\xi_{i}(t)=\left(x_{i}^{T}(t), v_{i}^{T}(t)\right)^{T}, i \in \underline{M}$

$$
A=\left[\begin{array}{cc}
0_{n \times n} & I_{n \times n} \\
0_{n \times n} & k I_{n \times n}
\end{array}\right], \quad B=\left[\begin{array}{cc}
0_{n \times n} & 0_{n \times n} \\
I_{n \times n} & 0_{n \times n}
\end{array}\right] .
$$

Furthermore, let $\xi(t)=\left(\xi_{1}^{T}(t), \mathrm{L}, \xi_{M}^{T}(t)\right)^{T}$, then the dynamic network is of the following form

$$
\xi(t)=\left(I_{M} \otimes A\right) \xi(t)-(L \otimes B) \xi(t-\tau(t))
$$

where $L$ is the Laplacian associated with the connected graph $G$. Moreover, we have the following result, which is similar to the dynamic systems without time- delay [10].

Lemma 2 The dynamics of System 7 is stabilized if and only if $M$ systems

$$
\eta_{i} \alpha(t)=A \eta_{i}(t)-\lambda_{i} B \eta_{i}(t-\tau(t))
$$

are globally asymptotical stable, where $\lambda_{i}, i \in \underline{M}$ are the nonnegative eigenvalues of $L$. 
Proof Since the Laplacian $L$ of undirected graph $G$ is real symmetric matrix, there exists an orthogonal matrix $W$ such that

$$
W^{T} L W=\Lambda=\left[\begin{array}{cccc}
\lambda_{1} & 0 & \mathrm{~L} & 0 \\
0 & \lambda_{2} & \mathrm{~L} & 0 \\
\mathrm{M} & \mathrm{M} & \mathrm{O} & \mathrm{M} \\
0 & 0 & \mathrm{~L} & \lambda_{M}
\end{array}\right]
$$

where $\lambda_{i}, i \in \underline{M}$ are the nonnegative real eigenvalues of Laplacian $L$. By the transform $\eta=\left(W \otimes I_{2 n \times 2 n}\right) \xi$, we may obtain

$$
\begin{aligned}
\eta \propto & =\left(W \otimes I_{2 n \times 2 n}\right)\left[\left(I_{M} \otimes A\right) \xi(t)-(L \otimes B) \xi(t-\tau(t))\right] \\
& =\left(I_{M} \otimes A\right) \eta(t)-\Lambda \eta(t-\tau(t))
\end{aligned}
$$

which implies that the dynamics of System 7 is stabilized if and only if $M$ Systems 8 are globally asymptotical stable.

\subsection{Main Results}

First, by means of linear matrix inequality (LMI), we study consensus stability of dynamic Systems 8 with certain communication transmission time-varying delay $\tau(t)$.

Theorem 1 The dynamic equations of (8) that the eigenvalues of Laplacian $L$ are zero, i.e., $\lambda_{s}=0$ for some $s \in \underline{M}$, achieves globally asymptotical stable if the control gain $k<0$.

Moreover, let $\eta_{s}(t)=\left(\begin{array}{l}\eta_{1 s}(t) \\ \eta_{2 s}(t)\end{array}\right) \otimes 1_{n}$, then

$$
\lim _{t \rightarrow \infty} \eta_{s}(t)=\left(\begin{array}{c}
x^{*} \\
0
\end{array}\right) \otimes 1_{n},
$$

where $x^{*}=\eta_{s 1}(0)-\frac{1}{k} \eta_{s 2}(0)$.

Proof Consider the dynamic equations of (8) with $\lambda_{s}=0$ for some $s \in \underline{M}$, it is easy to obtain their expressions as the following

$\eta_{s} \mathcal{s}_{s}(t)=A \eta_{s}(t)$.

Denoting $\eta_{s}(t)=\left(\begin{array}{l}\eta_{1 s}(t) \\ \eta_{2 s}(t)\end{array}\right) \otimes 1_{n}$, we have

$$
\eta_{s 1}(t)=\eta_{s 2}(t), \quad \eta_{s 2}(t)=k \eta_{s 2}(t) .
$$

Then

$$
\eta_{s 2}(t)=e^{k t} \eta_{s 2}(0), \eta_{s 2}(t)=\frac{e^{k t}-1}{k} \eta_{s 2}(0)+\eta_{s 1}(0) .
$$

Since $k<0$, one gets

$$
\lim _{t \rightarrow \infty} \eta_{s 1}(t)=\eta_{s 1}(0)-\frac{1}{k} \eta_{s 2}(0), \lim _{t \rightarrow \infty} \eta_{s 2}(t)=0 .
$$

which leads to the result of Theorem 1.
In order to prove our main result relevant to the dynamic Systems 8 with communication transmission time-varying delay, we recite the following lemma [7].

Lemma 3 For any real differentiable vector function $z(t) \in R^{n}$ and any $n \times n$ symmetric positive definite matrix $\Gamma$, one has the following inequality

$$
\begin{aligned}
& {[z(t)-z(t-\tau(t))]^{T} \cdot \Gamma \cdot[z(t)-z(t-\tau(t))]} \\
& \leq d \int_{t-\tau(t)}^{t}(s)^{T} \Gamma(s) d s
\end{aligned}
$$

where $\tau(t)$ satisfies $0 \leq \tau(t) \leq d$.

Theorem 2 Assume that the control gain $k<0$ and the communication transmission time-varying delay satisfies (3). If there exist symmetric positive definite matrices $P_{i}, Q_{i}, R_{i} \in R^{2 n \times 2 n}$ such that the following conditions hold:

$$
\left[\begin{array}{ll}
\Phi_{i 1} & \Phi_{i 2} \\
\Phi_{i 2}^{T} & \Phi_{i 3}
\end{array}\right]<0, \quad \Phi_{i 3}>0
$$

where

$$
\begin{aligned}
& \Phi_{i 1}=-\Omega_{i}+h Q_{i}+d\left(A-\lambda_{i} B\right)^{T} R_{i}\left(A-\lambda_{i} B\right), \\
& \Phi_{i 2}=\lambda_{i} P_{i} B+(1-h) Q_{i}+d \lambda_{i}\left(A-\lambda_{i} B\right)^{T} R_{i} B, \\
& \Phi_{i 3}=\frac{1}{d} R_{i}+(1-h) Q_{i}-d \lambda_{i}^{2} B^{T} R_{i} B, \\
& \Omega_{i}=-\left[\left(A-\lambda_{i} B\right)^{T} P_{i}+P_{i}\left(A-\lambda_{i} B\right)\right] .
\end{aligned}
$$

with properly choosing $d \geq 0, h \geq 0$. Then the origin of the $i$-th dynamic System 8 is asymptotical stable equilibrium point if and only if the communication networked topology $G$ is connected.

Proof (Sufficiency) Since the undirected communication networked topology $G$ is connected, the eigenvalues $\lambda_{i}, i=2, \mathrm{~L}, M$ of Laplacian $L$ are positive numbers in addition to $\lambda_{1}=0$ from Lemma 1. Consider the characteristic polynomial of $A-\lambda_{i} B, i=2, \mathrm{~L}, M$

$$
\pi_{A-\lambda_{i} B}(s)=\operatorname{det}\left(s I-\left(A-\lambda_{i} B\right)\right)=\left(s^{2}-k s+\lambda_{i}\right)^{n} .
$$

Since $k<0$ and $\lambda_{i}>0$, it is easy to be verify that $A-\lambda_{i} B$ is Hurwitz. Then there exists a symmetric positive definite matrix $P_{i}$ such that

$\Omega_{i}=-\left[\left(A-\lambda_{i} B\right)^{T} P_{i}+P_{i}\left(A-\lambda_{i} B\right)\right]$ is positive definite matrix. So (10) is always feasible for appropriate positive scalars $h$ and $d$.

Take a Lyapunov function for the $i$-th dynamic System 8 as follows:

$$
\begin{aligned}
V_{i}(t)= & \eta_{i}^{T}(t) P_{i} \eta_{i}(t)+\int_{t-\tau(t)}^{t} \eta_{i}^{T}(s) Q_{i} \eta_{i}(s) d s \\
& +\int_{-d}^{0}(s+d) \eta_{i}^{T}(t+s) R_{i} \eta_{1}(t+s) d s
\end{aligned}
$$

Rewrite the $i$-th dynamic System 8 as the following equivalent form 


$$
\eta_{i} \oint_{i}(t)=\left(A-\lambda_{i} B\right) \eta_{i}(t)+\lambda_{i} B \zeta_{i}(t)
$$

where $\zeta_{i}(t)=\eta_{i}(t)-\eta_{i}(t-\tau(t))$. Along the trajectory of the solution of System 12, we have

$$
\begin{aligned}
& L_{i}(t)=\eta_{i}^{T}(t)\left[\left(A-\lambda_{i} B\right)^{T} P_{i}+P_{i}\left(A-\lambda_{i} B\right)\right] \eta_{i}(t) \\
& +\lambda_{i}\left[\eta_{i}^{T}(t) P_{i} B \zeta_{i}(t)+\zeta_{i}^{T}(t) B^{T} P_{i} \eta_{i}(t)\right] \\
& +\eta_{i}^{T}(t) Q_{i} \eta_{i}(t)-(1-\tau(t)) \eta_{i}^{T}(t-\tau(t)) Q_{i} \eta_{i}(t-\tau(t)) \\
& +d r_{t} \&(t) R_{i} \eta_{t}\left\{(t)-\int_{-d}^{0} n_{t} \&(t+s) R_{i} \eta \psi_{t}(t+s) d s .\right.
\end{aligned}
$$

With the condition (3) and Lemma 2, we have

$$
\begin{aligned}
& \mathcal{S}_{i}(t) \leq \eta_{i}^{T}(t)\left[\left(A-\lambda_{i} B\right)^{T} P_{i}+P_{i}\left(A-\lambda_{i} B\right)+h Q_{i}\right. \\
& +d\left(A-\lambda_{i} B\right)^{T} R_{i}\left(A-\lambda_{i} B\right) \eta_{i}(t)+\eta_{i}^{T}(t)\left[\lambda_{i} P_{i} B\right. \\
& \left.+(1-h) Q_{i}+d \lambda_{i}\left(A-\lambda_{i} B\right)^{T} R_{i} B\right] \zeta_{i}(t)+\zeta_{i}^{T}(t) \\
& \cdot\left[\lambda_{i} B^{T} P_{i}+(1-h) Q_{i}+d \lambda_{i} B^{T} R_{i}\left(A-\lambda_{i} B\right)\right] \cdot \eta_{i}(t) \\
& -\zeta_{i}^{T}(t)\left[(1-h) Q_{i}-d \lambda_{i}^{2} B^{T} R_{i} B+d^{-1} R_{i}\right] \zeta_{i}(t) \\
& =\left(\eta_{i}^{T}(t) \quad \zeta_{i}^{T}(t)\right)\left[\begin{array}{cc}
\Phi_{i 1} & \Phi_{i 2} \\
\Phi_{i 2}^{T} & -\Phi_{i 3}
\end{array}\right]\left(\begin{array}{l}
\eta_{i}(t) \\
\zeta_{i}(t)
\end{array}\right)
\end{aligned}
$$

where $\Phi_{i j}, j=1,2,3$ are defined in (10). Therefore, there exists a positive constant $\beta_{i}$ such that

$$
\nu_{i}^{\&}(t) \leq-\beta_{i}\left\|\begin{array}{l}
\eta_{i}(t) \\
\zeta_{i}(t)
\end{array}\right\| \leq-\beta_{i}\left\|\eta_{i}(t)\right\| .
$$

This implies that the $i$-th dynamic System of 8 achieve asymptotical stable for $0 \leq \tau(t) \leq d$ and $0 \leq \tau(t) \leq h$.

(Necessary) Since the origin of the dynamic Systems 8 is asymptotical stable equilibrium point, the eigenvalue of $A-\lambda_{i} B$ have negative real-parts except that at most $n$ eigenvalues are zero. Considering the Laplacian $L$ and the characteristic polynomial of $A-\lambda_{i} B$, one may get $\lambda_{1}=0$ and the eigenvalues $\lambda_{i}, i=2, \mathrm{~L}, M$ of Laplacian $L$ are positive numbers. By Lemma 1, we may get the communication networked topology is connected.

Due to the reversible orthogonal transform, the $M$ dynamic Systems 8 are equivalent to the System 7. So we get the same result of stability for the System 7 .

Theorem 3 Assume that the graph $G$ is connected, the control gain $k<0$ and the communication transmission time-varying delay satisfies (3). If there exist symmetric positive definite matrices $P_{i}, Q_{i}, R_{i} \in R^{2 n \times 2 n}$ such that the following linear matrix inequalities hold

$$
\left[\begin{array}{ccccc}
\Phi_{21} & \Phi_{22} & \mathrm{~L} & 0_{2 n \times 2 n} & 0_{2 n \times 2 n} \\
\Phi_{22}^{T} & -\Phi_{23} & \mathrm{~L} & 0_{2 n \times 2 n} & 0_{2 n \times 2 n} \\
\mathrm{M} & \mathrm{M} & \mathrm{O} & \mathrm{M} & \mathrm{M} \\
0_{2 n \times 2 n} & 0_{2 n \times 2 n} & \mathrm{~L} & \Phi_{M 1} & \Phi_{M 2} \\
0_{2 n \times 2 n} & 0_{2 n \times 2 n} & \mathrm{~L} & \Phi_{M 2}^{T} & -\Phi_{M 3}
\end{array}\right]<0
$$

$$
\Phi_{i 3}>0
$$

with properly choosing positive scalars $h$ and $d$, where $\Phi_{i j}(i \in\{2, \mathrm{~L}, M\}$ and $j=1,2,3)$ are defined in (11). Then the dynamic System 7 achieves globally asymptotical consensus stability if and only if the communication networked topology $G$ is connected.

Moreover,

$$
\lim _{t \rightarrow \infty} \xi(t)=1_{M} \otimes\left(\begin{array}{l}
\xi^{*} \\
0
\end{array}\right) \otimes 1_{n},
$$

where $\xi^{*}=\frac{1}{M} \sum_{i=1}^{M}\left[x_{i}(0)-\frac{1}{k} v_{i}(0)\right] \otimes 1_{n}$.

Proof By Lemma 1, the networked topology $G$ is connected if and only if the real eigenvalues of Laplacian $L$ with $0=\lambda_{1}<\lambda_{2} \leq \lambda_{3} \leq L \leq \lambda_{M}$. Under the given conditions, the $M$ dynamic Systems 8 achieve globally asymptotical stable if and only if the communication networked topology $G$ is connected from Theorem 1 and Theorem 2.

Since the transform $\eta=\left(W \otimes I_{2 n \times 2 n}\right) \xi$ is reversible orthogonal, the $M$ dynamic Systems 8 are equivalent to System 7. Hence, for appropriate positive scalars $h$ and $d$, one can conclude that the System 7 achieves globally asymptotically consensus stability if and only if the communication networked topology $G$ is connected. And the allowable $h$ and $d$ can be obtained by the feasible linear matrix Inequality 13.

As the origin of the dynamic Systems of 8 for $i \in\{2, \mathrm{~L}, M\}$ is asymptotically stable equilibrium, we have $\left(\eta_{2}^{T}(t) \quad \mathrm{L} \quad \eta_{M}^{T}(t)\right) \rightarrow 0_{1 \times 2 n(M-1)}$.

Due to the fact that one eigenvalue of Laplacian is zero with eigenvector $1_{M}=(1, \mathrm{~L}, 1)^{T}$, one may get

$$
W=\frac{1}{\sqrt{M}}\left[\begin{array}{cc}
1 & 1_{M-1}^{T} \\
1_{M-1} & W_{1}
\end{array}\right]
$$

with $W_{1} 1_{M-1}=-1_{M-1}, \quad W_{1}^{T} W_{1}=M I_{M-1}-1_{M-1} 1_{M-1}^{T}$.

By Lemma $\backslash$ ref $\{$ lem1 $\}$ and Lemma 2, one gets

$$
\begin{aligned}
\left(\eta_{2}^{T},\right. & \left.\mathrm{L}, \quad \eta_{M}^{T}\right)^{T}=\frac{1}{\sqrt{M}}\left(1_{M-1} \otimes I_{2 n \otimes 2 n}\right) \xi_{1} \\
& +\frac{1}{\sqrt{M}}\left(W_{1}^{T} \otimes I_{2 n \otimes 2 n}\right)\left(\xi_{2}^{T}, \quad \mathrm{~L}, \quad \xi_{M}^{T}\right)^{T}
\end{aligned}
$$

Then, we can obtain that

$$
\xi_{i}=\bar{\xi}=\frac{1}{M} \sum_{m=1}^{M} \xi_{m}, \quad i \in\{2, \mathrm{~L}, M\} .
$$

One can get $\eta_{1}=\frac{1}{\sqrt{M}} \sum_{i=1}^{M} \xi_{i}$ by Lemma 1 . Then from Theorem 1, it is hold that $\lim _{t \rightarrow \infty} \eta_{1}(t)=x^{*}\left(\begin{array}{l}1_{n} \\ 0\end{array}\right)$, with 


$$
x^{*}=\eta_{11}(0)-\frac{1}{k} \eta_{12}(0)=\frac{1}{\sqrt{M}} \sum_{i=1}^{M}\left[x_{i}(0)-\frac{1}{k} v_{i}(0)\right] .
$$

Therefore, we can obtain

$$
\begin{gathered}
\lim _{t \rightarrow \infty} x_{i}(t)=\frac{1}{M} \sum_{i=1}^{M}\left[x_{i}(0)-\frac{1}{k} v_{i}(0)\right] \otimes 1_{n}, \\
\lim _{t \rightarrow \infty} v_{i}(t)=0_{n \times 1}
\end{gathered}
$$

The proof of the Theorem is completed.

Remark For any $h \geq 0$, the maximal allowable $d$ guaranteeing average consensus in Theorem 2 and/or Theorem 3 can be obtained from the following optimization problem:

Maximize $d$

s.t. $0 \leq h<1, P_{i}>0, Q_{i}>0, \quad R_{i}>0$ and (13).

This optimization problem can be solved by using the GEVP solver in Matlab's Control Systems Toolbox [5].

Considering the matrices in the linear matrix Inequality 13 are continuous for $\lambda_{i}>0, i \in\{2, \mathrm{~L}, M\}$, we may obtain the following corollary for estimation of conservative upper bound $h$ and $d$.

Corollary 1. Assume that the control gain $k<0$ and the communication time-varying delay satisfies (3). If there exist symmetric positive definite matrices $P_{i}, Q_{i}, R_{i} \in R^{2 n \times 2 n}$ such that the following linear matrix inequalities hold

$$
\begin{aligned}
& {\left[\begin{array}{cccc}
\Phi_{21} & \Phi_{22} & 0_{2 n \times 2 n} & 0_{2 n \times 2 n} \\
\Phi_{22}^{T} & -\Phi_{23} & 0_{2 n \times 2 n} & 0_{2 n \times 2 n} \\
0_{2 n \times 2 n} & 0_{2 n \times 2 n} & \Phi_{M 1} & \Phi_{M 2} \\
0_{2 n \times 2 n} & 0_{2 n \times 2 n} & \Phi_{M 2}^{T} & -\Phi_{M 3}
\end{array}\right]<0} \\
& \Phi_{23}>0 \quad \Phi_{M 3}>0
\end{aligned}
$$

with properly choosing $d \geq 0$ and $h \geq 0$, where $\Phi_{2 j}$, $\Phi_{M j}(j=1,2,3)$ are defined in (13). Then the dynamic System 7 achieves globally asymptotical consensus stability if and only if the communication networked topology $G$ is connected.

When time-varying delays satisfy (4), that is to say, nothing has been known about the derivative of $\tau(t)$. For Systems 8, one may construct the following Lyapunov function as

$$
W_{i}(t)=\eta_{i}^{T}(t) S_{i} \eta_{i}(t)+\int_{-d}^{0} \int_{t+\theta}^{t} \eta_{i}^{T}(s) R_{i} \eta_{i}(s) d s d \theta .
$$

Similar to the proof of Theorem 2 and Theorem 3, it is easy to get the following results and we may omit their proof here.

Theorem 4 Assume that the control gain $k<0$ and the communication transmission time-varying delay satisfies (4). If there exist symmetric positive definite matrices $S_{i}, T_{i} \in R^{2 n \times 2 n}$ such that the following conditions hold:

$$
\left[\begin{array}{ll}
\Psi_{i 1} & \Psi_{i 2} \\
\Psi_{i 2}^{T} & \Psi_{i 3}
\end{array}\right]<0, \quad \Psi_{i 3}>0
$$

where

$$
\begin{aligned}
& \Psi_{i 1}=-\Gamma_{i}+d\left(A-\lambda_{i} B\right)^{T} T_{i}\left(A-\lambda_{i} B\right), \\
& \Psi_{i 2}=\lambda_{i} S_{i} B+d \lambda_{i}\left(A-\lambda_{i} B\right)^{T} T_{i} B, \\
& \Psi_{i 3}=\frac{1}{d} T_{i}-d \lambda_{i}^{2} B^{T} T_{i} B, \\
& \Gamma_{i}=-\left[\left(A-\lambda_{i} B\right)^{T} S_{i}+S_{i}\left(A-\lambda_{i} B\right)\right] .
\end{aligned}
$$

with properly choosing $d \geq 0$. Then the origin of the $i$-th dynamic System of 8 is asymptotical stable equilibrium point if and only if the communication networked topology $G$ is connected.

Theorem 5 Assume that the graph $G$ is connected, the control gain $k<0$ and the communication transmission time-varying delay satisfies (4). If there exist symmetric positive definite matrices $S_{i}, T_{i} \in R^{2 n \times 2 n}$ such that the following linear matrix inequalities hold

$$
\left[\begin{array}{ccccc}
\Psi_{21} & \Psi_{22} & \mathrm{~L} & 0_{2 n \times 2 n} & 0_{2 n \times 2 n} \\
\Psi_{22}^{T} & -\Psi_{23} & \mathrm{~L} & 0_{2 n \times 2 n} & 0_{2 n \times 2 n} \\
\mathrm{M} & \mathrm{M} & \mathrm{O} & \mathrm{M} & \mathrm{M} \\
0_{2 n \times 2 n} & 0_{2 n \times 2 n} & \mathrm{~L} & \Psi_{M 1} & \Psi_{M 2} \\
0_{2 n \times 2 n} & 0_{2 n \times 2 n} & \mathrm{~L} & \Psi_{M 2}^{T} & -\Psi_{M 3}
\end{array}\right]<0
$$

with properly choosing positive scalars $d$, where $\Psi_{i j}$ ( $i \in\{2, \mathrm{~L}, M\}$ and $j=1,2,3$ ) are defined in (17).

Then the dynamic System 7 achieves globally asymptotical consensus stability if and only if the communication networked topology $G$ is connected.

Moreover,

$$
\lim _{t \rightarrow \infty} \xi(t)=1_{M} \otimes\left(\begin{array}{l}
\xi^{*} \\
0
\end{array}\right) \otimes 1_{n}
$$

where $\xi^{*}=\frac{1}{M} \sum_{i=1}^{M}\left[x_{i}(0)-\frac{1}{k} v_{i}(0)\right] \otimes 1_{n}$.

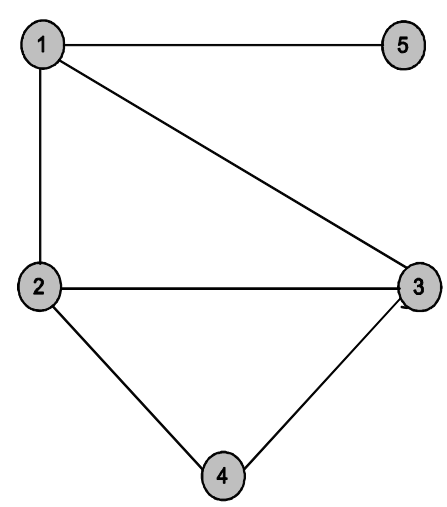

Figure 1 Undirected connected graph $G$ with five nodes. 


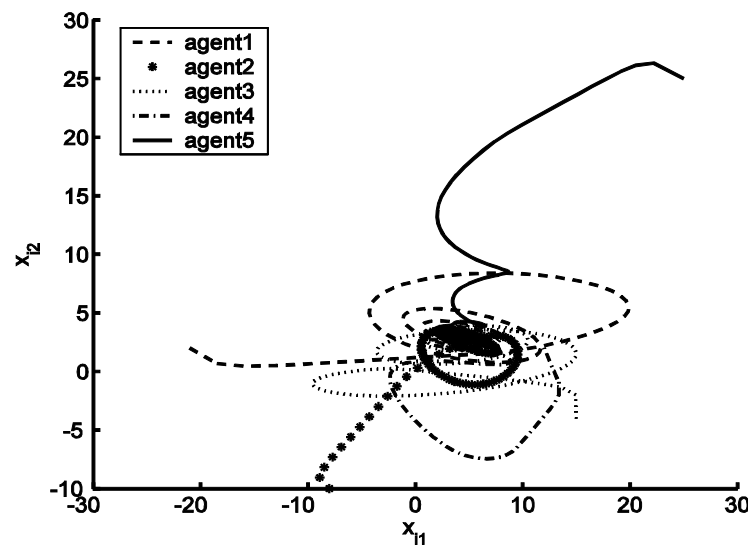

Figure 2. State trajectories of the agents in $G$.

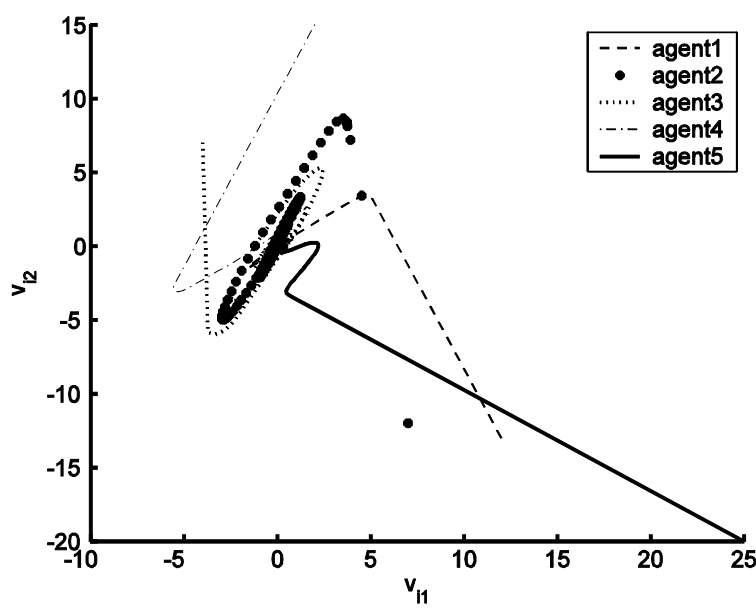

Figure 3. Velocity trajectories of the agents in $G$.

\section{Simulations}

Numerical simulations will be given to illustrate the theoretical results obtained in the previous section. Consider five dynamic agents under network described in Figure 1.

Here we consider the dynamical equations ( $\operatorname{ref}\{\mathrm{dyn} 0\}$ ) with $n=2$. By employing the LMI Toolbox in Matlab, one gets that the maximum time-delay bound is $d=2.1152$ when $h=0$, i.e. the value of time-delay is fixed. When $h=0.5$, the maximum delay bound is $d=1.2799$. And we may get the corresponding feasible solutions in the following.

$P_{1}=\left[\begin{array}{ll}1.4978 I_{2} & 0.2519 I_{2} \\ 0.2519 I_{2} & 0.9299 I_{2}\end{array}\right] \quad P_{2}=\left[\begin{array}{ll}1.3969 I_{2} & 0.2175 I_{2} \\ 0.2175 I_{2} & 0.6666 I_{2}\end{array}\right]$

$P_{3}=\left[\begin{array}{ll}1.2385 I_{2} & 0.0985 I_{2} \\ 0.0985 I_{2} & 0.2196 I_{2}\end{array}\right] P_{4}=\left[\begin{array}{ll}1.6896 I_{2} & 0.5056 I_{2} \\ 0.2056 I_{2} & 0.2557 I_{2}\end{array}\right]$

$Q_{1}=\left[\begin{array}{ll}0.1784 I_{2} & 0.0334 I_{2} \\ 0.0334 I_{2} & 0.6915 I_{2}\end{array}\right] Q_{2}=\left[\begin{array}{ll}0.3766 I_{2} & 0.0488 I_{2} \\ 0.0488 I_{2} & 0.6925 I_{2}\end{array}\right]$

$$
\begin{aligned}
& R_{3}=\left[\begin{array}{ll}
2.0632 I_{2} & 0.1171 I_{2} \\
0.1171 I_{2} & 0.0099 I_{2}
\end{array}\right] R_{4}=\left[\begin{array}{cc}
2.4174 I_{2} & 0.1864 I_{2} \\
0.1864 I_{2} & 0.0471 I_{2}
\end{array}\right] \\
& Q_{3}=\left[\begin{array}{cc}
0.2463 I_{2} & -0.0197 I_{2} \\
-0.0197 I_{2} & 0.3858 I_{2}
\end{array}\right] Q_{4}=\left[\begin{array}{cc}
0.8508 I_{2} & -0.1547 I_{2} \\
-0.1547 I_{2} & 0.0829 I_{2}
\end{array}\right] \\
& R_{1}=\left[\begin{array}{cc}
1.4655 I_{2} & -0.0197 I_{2} \\
-0.0197 I_{2} & 0.1397 I_{2}
\end{array}\right] R_{2}=\left[\begin{array}{cc}
1.9895 I_{2} & -0.0281 I_{2} \\
-0.0281 I_{2} & 0.0714 I_{2}
\end{array}\right]
\end{aligned}
$$

The agents have initial conditions $x_{1}(\theta)=\left(\begin{array}{ll}-21 & 2\end{array}\right)^{T}$, $x_{2}(\theta)=\left(\begin{array}{ll}-8 & -10\end{array}\right)^{T}, \quad x_{3}(\theta)=\left(\begin{array}{ll}-15 & 4\end{array}\right)^{T}, \quad x_{4}(\theta)=\left(\begin{array}{ll}12 & 2\end{array}\right)^{T}$, $x_{5}(\theta)=\left(\begin{array}{ll}25 & 25\end{array}\right)^{T}, \quad v_{1}(\theta)=\left(\begin{array}{lll}12 & -13\end{array}\right)^{T}, \quad v_{2}(\theta)=\left(\begin{array}{ll}-12 & 5\end{array}\right)^{T}$, $v_{3}(\theta)=\left(\begin{array}{ll}7 & 18\end{array}\right)^{T}, \quad v_{4}(\theta)=\left(\begin{array}{ll}15 & -25\end{array}\right)^{T}, \quad v_{5}(\theta)=\left(\begin{array}{ll}-20 & 15\end{array}\right)^{T}$ for $\theta \in[-1,0]$. The eigenvalues of the Laplacian matrix are $\lambda_{1}=0, \quad \lambda_{2}=0.8299, \quad \lambda_{3}=2.6889, \quad \lambda_{4}=4$, $\lambda_{5}=4.4812$. Figure 2 and Figure 3 show the state and velocity trajectories of the multi-agent systems with time-varying delay $\tau(t)=0.2|\sin t|$.

\section{Conclusions}

In this paper, we discuss the coordinate stability of multi-agent systems where the agent is described by double-integrator with time-varying transmission delay in their communicated network. Two different time-varying delays are considered for dynamical systems. We firstly decompose the multi-agent systems into \$M dynamical systems by certain transformation of state space under the condition of undirected connected communication network. By the methods of linear matrix inequality (LMI), we study each dynamical system with time-varying delay and show that the agents of multi-agent systems can achieve globally asymptotical consensus stability. Meanwhile, the upper bound parameters of time-varying delay can be estimated by checking solutions of LMI. Numerical simulation results are provided and demonstrate the effectiveness of our theoretical results.

\section{Acknowledgment}

This work is supported by National Nature Science Foundation of China under Grant 60674046, the Nanjing Audit University Scientific Research Start-up Fund for High-level Talents and the Theory and Application of Differential Equations Foundation of the Nanjing Audit University.

\section{References}

[1] A. Jadbabaie, J. Lin, and S. A. Morse, "Coordination of groups of mobile autonomous agents using nearest neighbor rules," IEEE Transactions on Automatic Control, Vol. 48, No. 6, pp. 988-1000, June 2003. 
[2] W. Ren and R. W. Beard, "Consensus seeking in multiagent systems under dynamically changing interaction topologies," IEEE Transactions on Automatic Control, Vol. 50, No. 5, pp. 655-661, May 2005.

[3] R. O. Saber and R. M. Murray, "Consensus problems in networks of agents with switching topology and timedelays," IEEE Transactions on Automatic Control, Vol. 49, No. 9, pp. 1520-1533, September 2004.

[4] R. O. Saber, J. A. Fax, and R. M. Murray, "Consensus and cooperation in networked multiagent systems," Proceedings of the IEEE, Vol. 95, No. 1, pp. 215-240, January 2007.

[5] L. Moreau, "Stability of multiagent systems with timedependent communication," IEEE Transactions on Automatic Control, Vol. 50, No. 2, pp. 169-182, February 2005 .

[6] D. Lee and W. M. Spong, "Agreement with non-uniform information delays," Proceedings of the 2006 American Control Conference, pp. 756-761, 2006.

[7] P. Lin and Y. Jia, "Average consensus in networks of multi-agents with both switching topology and coupling time-delay," Physica A, Vol. 387, pp. 303-313, 2008.

[8] Y. Sun, L. Wang, and G. Xie, "Average consensus in directed networks of dynamic agents with time-varying communication delays," Proceedings of the 45th IEEE Conference on Decision and Control, pp. 3393-3398, 2006.

[9] J. Hu and Y. Hong, "Leader-following coordination of multi-agent systems with coupling time delays," Physica A, Vol. 374, pp. 853-863, 2007.
[10] J. A. Fax and R. M. Murray, "Information flow and cooperative control of vehicle formations," IEEE Transactions on Automatic Control, Vol. 49, No. 9, pp. 1465 1476, 2004.

[11] G. Xie and L. Wang, "Information flow and cooperative control of vehicle formations," International Journal of Robust and Nonlinear Control, Vol. 17, pp. 941-959, 2007.

[12] L. Moreau, "Stability of continuous-time distributed consensus algorithms," In Proceedings of the 43rd IEEE Conference on Decision and Control, Atlantis, Paradise Island, Bahamas, pp. 3998-4003, 2004.

[13] Dongjun Lee and Mark W. Spong, "Agreement with non-uniform information delays", In Proceedings of the 2006 American Control Conference, Minnesota, USA, June 14-16, 2006.

[14] M. Cao, A. S. Morse, and B. D. O. Anderson, "Reaching a consensus using delayed information", In Proceedings of the 45th IEEE Conference on Decision and Control, San Diego, pp. 3375-3380, 2006.

[15] N. Biggs, "Algebraic, graph theory," Cambridge: Cambridge University Press, 1994.

[16] H. Yu, Y. Zheng, "Global behavior of dynamical agents in directed network," Proceedings of the 26th Chinese Control Conference, pp. 557-561, 2007.

[17] A. Isidori, "Nonlinear control systems II," London: Communications and Control Engineering Series, Springer-Verlag, 1999.

[18] R. A. Horn and C. R. Johnson, "Matrix analysis," Cambridge: Cambridge University Press, 1987. 